\title{
Stridor due to Crico-Arytenoid Fixation in Severe Reactive Arthritis: A Case Presentation Treated with CO2 Laser via a Laryngofissue
}

\section{Junaid Aamir ${ }^{1}$, Tudor Ionescu², Maha Khan²,3, Yakubu Karagama}

1. University of Manchester (corresponding author Junaidaamir@doctors.org.uk) 2. Department of Otolaryngology, Manchester University Foundation Trust 3. Health Education North West

\section{Introduction}

- Reactive arthritis is an inflammatory spondyloarthritis (SpA) that occurs following a gastrointestinal or genitourinary infection. ${ }^{[1]}$

- Around $18 \%$ of patients who suffer from reactive arthritis have been known to progress to a chronic polyarthritis. ${ }^{[2]}$

We present a case of a 51-year-old male suffering from reactive arthritis with otolaryngological manifestations

\section{Case Report}

\section{Patient Presentation}

- In January 2018, a 51 year-old male was admitted to hospital due to stridor following a bout of upper respiratory tract injection.

\section{Patient Background}

- The patient had a 33-year history of reactive arthritis.

- The arthritis had led to some significant complications and comorbidities leading to progressive diffuse joint stiffness and complex pain.

- Temporomandibular joint (TMJ) stiffness caused trismus of a severity which precluded orotracheal intubation.

\section{Investigations and Management}

- Flexible laryngoscopy revealed fixed arytenoids in adductor position.

- The patient underwent an emergency tracheostomy under a general anaesthesia.

- Our plan was to perform a CO2 laser partial arytenoidectomy and endoscopic lateralisation cordopexy.

- Microlaryngoscopy, however, was not possible due to TMJ stiffness.

- An external approach via laryngofissure, therefore, was considered.

\section{Surgical Approach}

- A $5 \mathrm{~cm}$ horizontal incision was performed in front of the thyroid cartilage.

- A $1 \mathrm{~mm}$ fissure burr was used to cut the thyroid cartilage vertically in the midline.

- A small access above the anterior commissure was established, sufficient enough to insert a 0-degree Hopkins rod, to visualise the entire larynx.

- A hand-held CO2 laser was used to perform a partial left arytenoidectomy.

- One lateralisation suture was placed to further lateralise the left vocal cord to create a wider posterior glottic gap; this suture was removed after 3 weeks to avoid a cheese-wire effect.

\section{Post Operative Period}

- Post-operatively, the patient suffered glottic oedema; he was kept as an inpatient and administered steroids regularly.

- Gradually, the swelling decreased and 2 months later he was decannulated.

- His voice following this was only minimally affected and there was no aspitation

References: 1. R. Terenzi, S. Monti, G. Tesei, and L. Carli, 'One year in review 2017: spondyloarthritis', Clin. Exp. Rheumatol., vol. 36, no. 1, pp. 1-14, Feb. 2018

2. T. Hannu, R. Inman, K. Granfors, and M. Leirisalo-Repo, 'Reactive arthritis or post-infectious arthritis?', Best Pract Res Clin Rheumatol, vol. 20, no. 3, pp. 419-433, Jun. 2006.

3. J. F. Daly and F. N. Kwok 'Laryngofissure and cordectomy', Laryngoscope, vol. 85, no. 8, pp. 1290-1297, Aug 1975
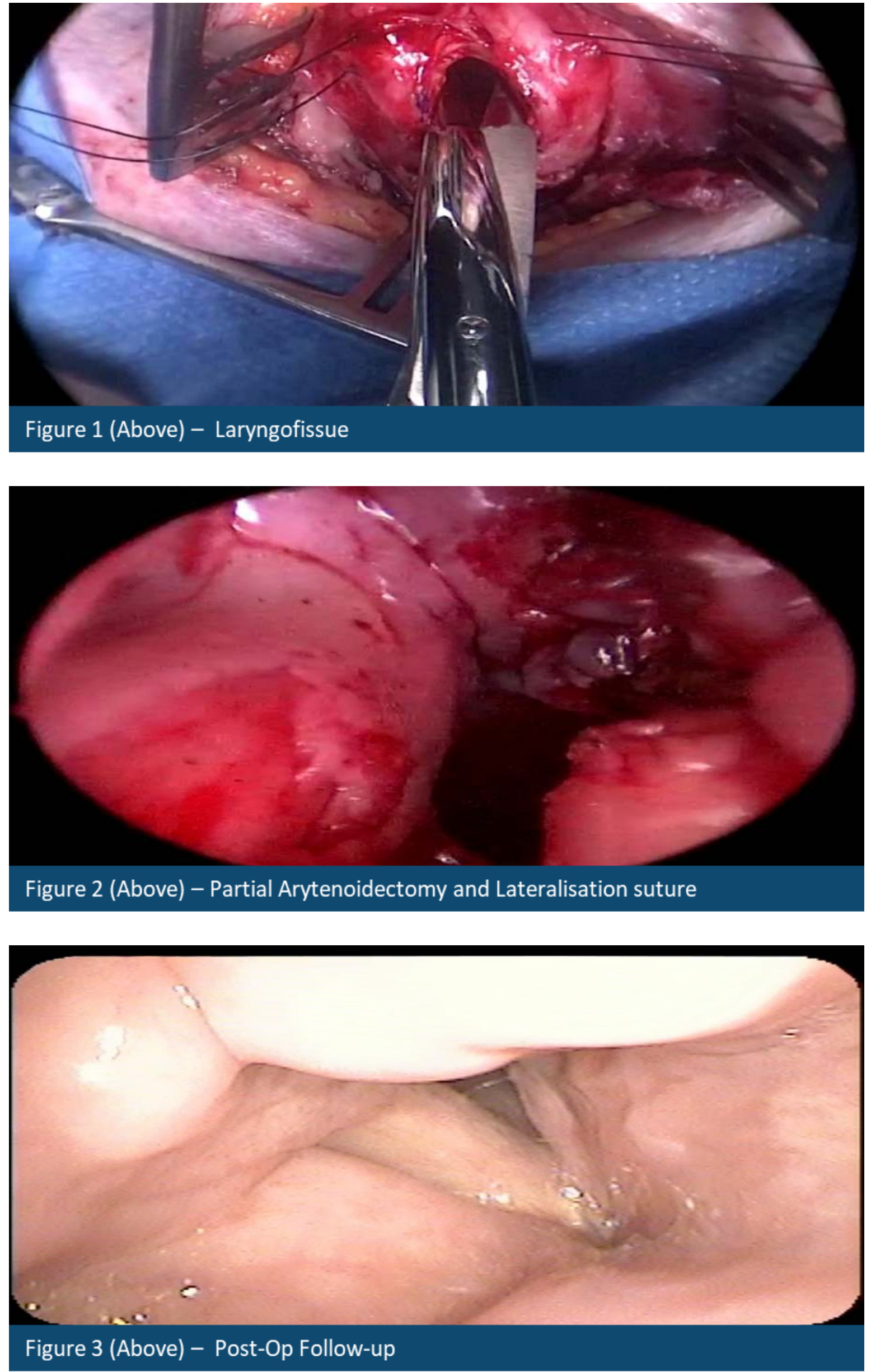

\section{Discussion}

- In our literature search, no case reports were found focusing on laryngeal involvement of patients suffering from reactive arthritis.

- Our focus in the management of this patient was to ensure that the patient's airway was appropriately managed whilst attempting to control the systemic disease.

- From past literature it was clear to see that the use of laryngofissure was in decline even as far back as 1975. ${ }^{[3]}$

- Alongside this, we found that there was very little literature regarding the use and efficacy of laryngofissure as a method to obtain access in the event of difficult access through the mouth.

\section{Conclusion}

- Cricoarytenoid arthritis (CAA) has been known to be a cause in acute airway obstruction, as seen in this case and others in the literature.

- Currently, the evidence regarding laryngeal involvement of SpA is longstanding but in need of further development.

- Laryngofissure is a key technique, that in the right situations, can be invaluable in obtaining access to a patient's larynx. 